www.jmscr.igmpublication.org

Index Copernicus Value: 79.54

ISSN (e)-2347-176x ISSN (p) 2455-0450

crossref DOI: https://dx.doi.org/10.18535/jmscr/v7i4.135

\title{
Study of Yield of Endoscopic Ultrasound: Fine Needle Aspiration Cytology in Patients with Solid Pancreatic Masses at S.M.S. Medical College, Jaipur
}

Authors

\section{Dr Murari Lal Meena ${ }^{1}$, Dr Dilip Ramrakhiani*, Dr Ashok Jhajharia ${ }^{3}$, Dr Rimjhim Shrimal ${ }^{4}$, Dr Ranjana Solanki ${ }^{5}$}

${ }^{1}$ Resident Doctor, Department of Pathology, ${ }^{2}$ Professor, Department of Pathology, ${ }^{3}$ Assistant Professor, Department of Gastroenterology, ${ }^{4}$ Senior Demonstrator, Department of Pathology, ${ }^{5}$ Senior Professor,

Department of Pathology

*Corresponding Author

Dr Dilip Ramrakhiani

Mobile No: 7737315766, Email: dilip.ramrakhiani@ gmail.com

\begin{abstract}
Solid masses of pancreas represent a variety of benign and malignant neoplasm of exocrine and endocrine tissue of pancreas. Endoscopic Ultrasound (EUS) is a relative new technology that employs endoscopic and high frequency ultrasound (US).
\end{abstract}

Keywords: EUS-FNAB, Pancreatic Carcinoma.

\section{Introduction}

Endoscopic Ultrasound (EUS) is relatively new technology that has been shown to be a highly sensitive method for the detection of pancreatic masses. ${ }^{[1]}$ It is more sensitive than conventional computed tomography (CT) scan for detecting small pancreatic tumors $(<3.0 \mathrm{~cm})$ and determining their resectability based on vessel invasion. ${ }^{[2]}$ Early detection is important as tumor size is an independent predictor of improved prognosis. ${ }^{[3]}$ EUS-guided fine-needle aspiration biopsy (EUSFNAB) is useful and accurate modality for characterizing lesions from the pancreas, lymph node, gastrointestinal tract wall, retro peritoneum, liver, biliary tree and adrenal glands. ${ }^{[4]}$ EUS-FNAB can be performed on small lesions, offering an opportunity for early detection of tumors, the staging of malignancies and in some instances, helping to avoid unnecessary surgeries.

The reported results of pancreatic EUS-FNA vary in the range of $64-95 \%$ for sensitivity, $75-100 \%$ for specificity and 78-95\% for diagnostic accuracy. ${ }^{[5]}$ Several factors can affect the results of EUS-FNA, such as the experience of the endo-sonographer, the position of the endoscope, the diameter of the needle, the number of passes, and the presence of an onsite cytopathologist. ${ }^{[6]}$ Furthermore, core biopsy specimens for assessing architectural features may be essential for diagnosing certain neoplasm, such as lymphomas and stromal cell tumors. ${ }^{[7]}$ However cost and staffing limitations frequently limit the availability $f$ an on-site cytopathologist at many centers. $^{[8]}$ 
Although EUS is highly sensitive in detecting pancreatic solid masses, its ability to differentiate between inflammatory masses and malignant disease is limited.1with the advent of curvilinear echo endoscope stransgastric and transduodenal EUS-FNAB of the pancreas have become a reality. ${ }^{[9]}$ EUS-with FNAB has become an important technique of gastroenterologists for the diagnosis of pancreatic adeno-carcinoma before chemotherapy and/ or surgery. EUS-FNAB, with its ability to obtain a tissue diagnosis, has increased the accuracy of EUS in the diagnosis of pancreatic adeno carcinoma. The diagnostic accuracy of EUS-FNAB was enhanced in prospective, multicenter studies and demonstrates that EUS-FNAB is a highly accurate diagnostic test for solid neoplasm of the pancreas. ${ }^{[10]}$ The survival rate of patients with these tumors is extremely poor, with an overall 5- year survival rate of less than 5\%, 12 making it one of the biggest "cancer killers". ${ }^{[13]}$ Therefore early and accurate diagnosis is vital for improving the efficacy of therapeutic intervention. In the current study, we prospectively evaluated the cellular yield of EUS-FNAB in patients with solid pancreatic masses who were clinically suspected to have pancreatic carcinoma. We also evaluated the clinical significance of anatypical or suspicious cytologic diagnosis and investigated the causes of falsenegative results with the aim to prospectively evaluate the yield of EUS-FNAB in the diagnosis of patients presenting with solid pancreatic lesion

\section{Material}

This descriptive type of prospective observational study conducted in Department of Pathology and Gastroenterology, S.M.S. Medical College Jaipur, Rajasthan from March 2017 to November 2018 in EUS-FNA specimens

Patients with solid pancreatic mass based on clinical results and/or other imaging studies Patients who required a tissue diagnosis or who failed other attempts by ERCP, CT-guided biopsy and/or USguided biopsy were included in the current study. Patients had previously undergone chemotherapy or radiotherapy and Cystic lesion of pancreas were excluded.

\section{Plan of Procedure}

$\mathrm{H}$ \& E staining, Wet fix smear, Ethyl-alcohol (Fixation) - 5 minutes, Dry slide, Hematoxyline 5-7 minutes, Running water 5-7 minutes, Eosin - 30 second, Acetone -3 jars ( 2 minutes in each jar), Xylene -2 jars (2 minutes in each jar), Dry mount Giemsa staining

Dry Smear, Air Dry, Methanol (fixation) 5 minutes, 1:10 (Giemsa stain: Distill water) mix pour on slide and rest for 20 minutes, Wash in running tap water till bluish colour appearance, Dry, Xylene dips - 2dip,Dry Mount

EUS-guided fine-needle aspiration of patients with solid pancreatic mass clinical/imaging was performed after exclusion criteria 80 Specimens was taken for analysis. Investigations applied staining done outcomes was analysed in terms of Proportion of - Malignant/ Suspicious for Malignancy/ Atypical/Benign.

Statistical analysis was performed with the SPSS, Trial version 23 for Windows statistical software package (SPSS inc., Chicago, il, USA) and Primer for the generation of descriptive and inferential statistics. The Categorical data were presented as numbers (percent). The quantitative data were presented as mean and standard deviation. The difference in proportion was analysed by using chi square test Statistical significance was set to $\mathrm{p}<$ 0.05 .

\section{Observation}

Most of the patients were in the age group of $51-$ 60 years $(43.8 \%)$ followed by $41-50$ years $(27.5 \%)$ and $61-70$ years $(21.3 \%)$. Only $3(3.7 \%)$ patients were below the age of 40 years. Also only $3(3.7 \%)$ patients were above the age of 70 years. The mean age of these patients with solid pancreatic mass was $55.48 \pm 8.96$ years ranging from 35 to 83 years. Most $(61.2 \%)$ of these patients were male and only 31 patients $(38.8 \%)$ were females.

Most of the female (45.2\%) as well as male (42.9\%) patients were in the age group of $51-60$ years 
followed by $41-50$ years $(32.3 \%$ of females and $24.5 \%$ of males). No significant difference was seen in the age distribution of pancreatic mass among male and female subjects ( $\mathrm{p}=0.538$ ).

EUS - FNAB suggested malignant lesion in most patients $(68.8 \%)$ while benign legion was suggested in $21(26.2 \%)$ patients. Four (5\%) patients had suspicious finding on EUS - FNAB. Final diagnosis was made on basis of the histopathology findings. Most of the patients $(83.8 \%)$ were finally diagnosed with malignant pancreatic mass and only $13(16.2 \%)$ were diagnosed with benign pancreatic mass. Of the 4 suspicious lesions 3 turned out to be malignant and only 1 was benign.

Out of the 67 patients with malignant mass, 55 $(82.1 \%)$ patients were correctly diagnosed by EUS FNAB as malignant, while $9(13.4 \%)$ were wrongly suggested as benign by EUS - FNAB and out of the 4 suspicious lesion 3 were malignant on final diagnosis. Out of 13 patients with benign pancreatic mass, $12(92.3 \%)$ were correctly diagnosed to have benign lesion and one patient had suspicious finding. Sensitivity of $86.57 \%$ indicates that $86.57 \%$ malignant cases are correctly diagnosed as malignant by EUS FNAB and only $13.43 \%$ of malignant cases were missed by EUS FNAB. Specificity was found to be $92.31 \%$ which indicates that most $(92.31 \%)$ of non-malignant cases were correctly excluded. i.e. $92.31 \%$ benign cases were correctly diagnosed as benign. A high PPV of $98.31 \%$ indicates that almost all cases suggested as malignant by EUS FNAB were finally diagnosed to be malignant. This is of great value for a clinician to immediately start treatment on positive finding on EUS FNAB. NPV was found to be low (57.14\%) i.e. among patients suggested to have benign lesion by EUS FNAB, only $57.14 \%$ were finally diagnosed to have benign lesion, and rest $42.86 \%$ were wrongly suggested as benign. This implicates that if EUS FNAB finding suggests benign lesion, the patients need to be cautiously followed up as there is $42.86 \%$ chance that the lesion may turn up to be malignant. The overall diagnostic accuracy of EUS FNAB was found to be $88.5 \%$ i.e. $88.5 \%$ of pancreatic masses are correctly classified as malignant / benign by
EUS FNAB adeno-carcinoma was the most common diagnosis in solid pancreatic masses found in $40(50 \%)$ of patients. Most common benign finding was chronic pancreatitis found in 21 (26.2\%) patients. Neuroendocrine carcinoma was found in 5 (6.3\%) patients. Malignant epithelial and poorly differentiated metastatic carcinoma were reported in $3(3.8 \%)$ pancreatic masses. Anaplastic giant cell carcinoma and spindle cell neoplasm were found in only 1 patient each. Suspicious for malignant finding on FNAC was reported in 4 patients.

Most of the benign pancreatic lesions were found in $51-60$ years $(46.1 \%)$ and $41-50$ years $(38.5 \%)$ age group. Most of the malignant pancreatic lesions were also found in $51-60$ years $(43.2 \%)$ and $41-$ 50 years $(25.4 \%)$. No significant difference was found in the age distribution of benign and malignant pancreatic lesions (0.754). Most of the benign pancreatic lesions were found in males $(76.1 \%)$. Malignant pancreatic lesions were found more in males (58.2\%) as compared to females (41.8\%). No significant difference was found in the gender distribution of benign and malignant pancreatic lesions $(\mathrm{p}=0.339)$.

Table 01: Age distribution of patients with solid pancreatic masses

\begin{tabular}{|l|c|c|}
\hline Age group (Years) & No. of subjects & Percentage \\
\hline $31-40$ years & 3 & 3.7 \\
\hline $41-50$ years & 22 & 27.5 \\
\hline $51-60$ years & 35 & 43.8 \\
\hline 61-70 years & 17 & 21.3 \\
\hline$>70$ years & 3 & 3.7 \\
\hline Total & $\mathbf{8 0}$ & $\mathbf{1 0 0}$ \\
\hline Gender & No. of subjects & Percentage \\
\hline Female & 31 & 38.8 \\
\hline Male & 49 & 61.2 \\
\hline Total & $\mathbf{8 0}$ & $\mathbf{1 0 0}$ \\
\hline EUS-FNAB finding & No. of subjects & Percentage \\
\hline Benign & 21 & 26.2 \\
\hline Malignant & 55 & 68.8 \\
\hline Suspicious & 4 & 5 \\
\hline Total & $\mathbf{8 0}$ & $\mathbf{1 0 0}$ \\
\hline Final diagnosis & No. of subjects & Percentage \\
\hline Benign & 13 & 16.2 \\
\hline Malignant & 67 & 83.8 \\
\hline Total & $\mathbf{8 0}$ & $\mathbf{1 0 0}$ \\
\hline
\end{tabular}


Table 02: Final diagnosis of solid pancreatic masses according to age of patients

\begin{tabular}{|l|c|c|c|c|c|c|c|}
\hline \multirow{2}{*}{$\begin{array}{l}\text { Age group } \\
\text { (years) }\end{array}$} & \multicolumn{2}{|c|}{ Benign } & \multicolumn{2}{c|}{ Malignant } & \multicolumn{2}{c|}{ Total } & \multirow{2}{*}{ P Value LS } \\
\cline { 1 - 7 } & $\mathbf{N}$ & $\mathbf{\%}$ & $\mathbf{N}$ & $\mathbf{\%}$ & $\mathbf{N}$ & $\mathbf{\%}$ & \\
\hline $31-40$ & 0 & 0 & 3 & 4.5 & 3 & 3.7 & \multirow{2}{*}{$0.754 \mathrm{NS}$} \\
\hline $41-50$ & 5 & 38.5 & 17 & 25.4 & 22 & 27.5 & \\
\hline $51-60$ & 6 & 46.1 & 29 & 43.2 & 35 & 43.8 & \\
\hline $61-70$ & 2 & 15.4 & 15 & 22.4 & 17 & 21.3 & \\
\hline$>70$ & 0 & 0 & 3 & 4.5 & 3 & 3.7 & \\
\hline Total & 13 & 100 & 67 & 100 & 80 & 100 & \\
\hline Female & 3 & 23.1 & 28 & 41.8 & 31 & 38.8 & \multirow{2}{*}{$0.33 \mathrm{NS}$} \\
\hline Male & 10 & 76.1 & 39 & 58.2 & 49 & 61.2 & \\
\hline Total & 13 & 100 & 67 & 100 & 80 & 100 & \\
\end{tabular}

Table 03: EUS-FNAB results in relation to final diagnosis of patients with solid pancreatic masses

\begin{tabular}{|l|c|c|c|c|}
\hline \multirow{2}{*}{$\begin{array}{l}\text { EUS-FNAB } \\
\text { finding }\end{array}$} & \multicolumn{2}{|c|}{ Final diagnosis } & \multirow{2}{*}{$\begin{array}{c}\text { Total } \\
\text { Sensitivity }\end{array}$} & $\begin{array}{c}\text { Diagnostic parameter } \\
\text { Value (95\% } \\
\text { confidence interval) }\end{array}$ \\
\cline { 2 - 3 } \cline { 5 - 5 } Malignant & Malignant & \multirow{2}{*}{ Benign } & & $\begin{array}{c}86.57 \% \\
(76.03-93.67)\end{array}$ \\
\hline Benign & $55(82.1 \%)$ & 0 & Specificity & $\begin{array}{c}92.31 \% \\
(63.97-99.81)\end{array}$ \\
\hline Suspicious & $9(13.4 \%)$ & $12(92.3 \%)$ & $\begin{array}{c}\text { Positive predictive } \\
\text { value }\end{array}$ & $\begin{array}{c}98.31 \% \\
(89.75-99.74)\end{array}$ \\
\hline Total & $3(4.5 \%)$ & $1(7.7 \%)$ & $\begin{array}{c}\text { Negative } \\
\text { predictive value }\end{array}$ & $\begin{array}{c}57.14 \% \\
(41.58-71.41)\end{array}$ \\
\hline
\end{tabular}

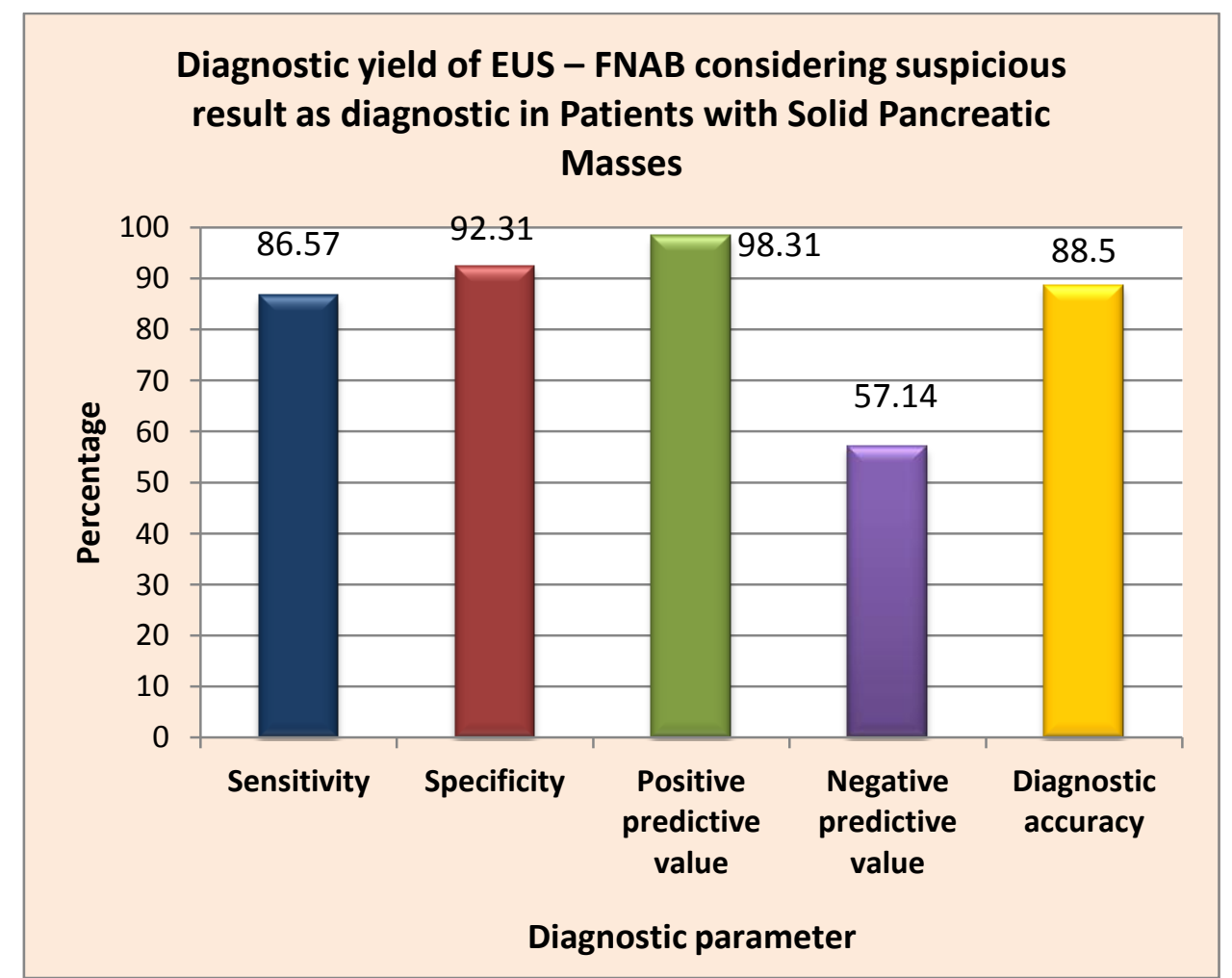

Fig. 1: Diagnostic Yield of EUS -FNAB considering suspicious result as diagnostic in patients with solid pancreatic masses 


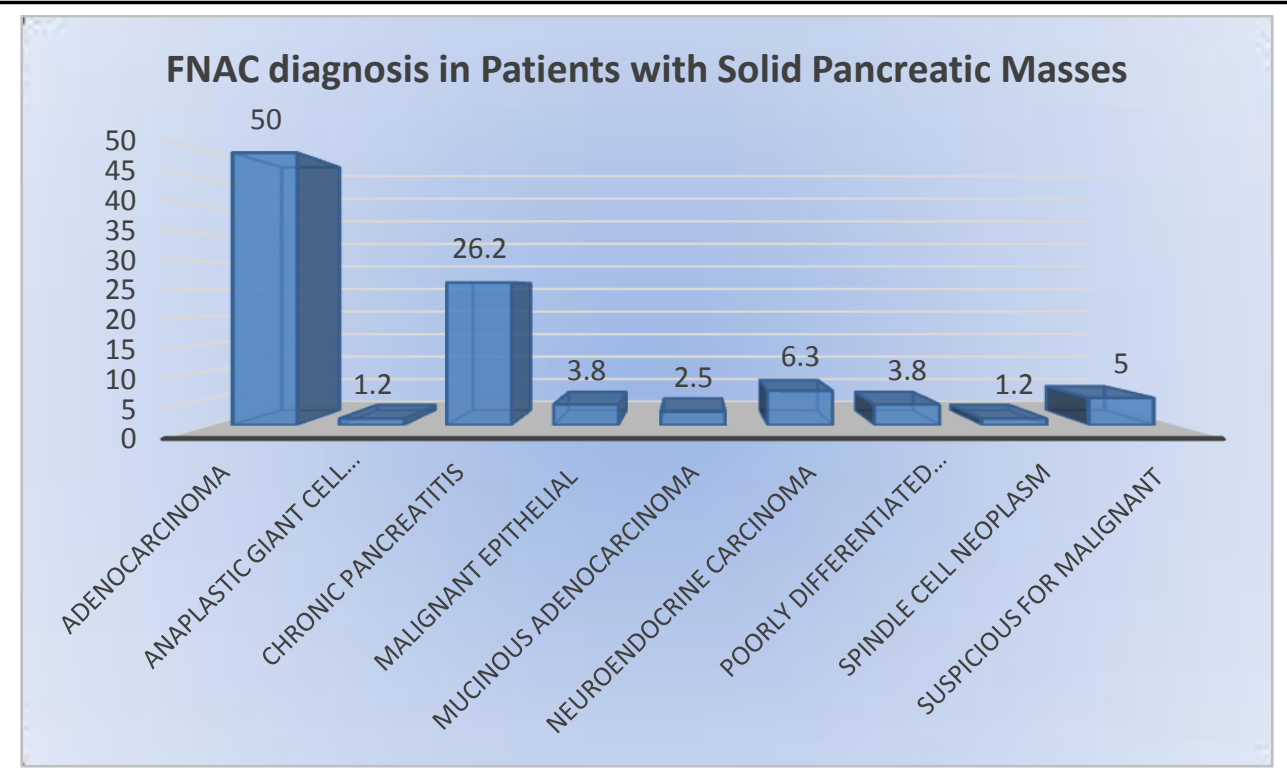

Fig. 2: Final diagnosis of Solid Pancreatic Masses

\section{Discussion}

This is laboratory based descriptive type of observational study conducted in the Department of Pathology and Gastroenterology, S.M.S. Medical College Jaipur, Rajasthan on EUS-FNA specimens received in Pathology Department of SMS Medical College Jaipur on 80 Patients with Solid Pancreatic Masses with the aim to contribute to the advancement of management for pancreatic cancer patients by improving the detection and diagnostic results.

In our current study, most of the patients were in the age group of 51 to 60 years $(43.8 \%)$ followed by 41 to 50 years $(27.5 \%)$ and then 61 to 70 years (21.3\%).

Only $3(3.7 \%)$ patients were below the age of 40 years and only $3(3.7 \%)$ patients were above the age of 70 years. The mean age of these patients with solid pancreatic mass was $55.48 \pm 8.96$ years ranging from 35 years to 83 years. This finding was similar with studies by Veronika Gagovic (2012)14 mean age of 66 years, Faming Zhang (2016)15with mean age $65.6 \pm 12.5$ years, Yang et al (2015)16mean age 61.8 \pm 11.4 years, Robert A et al (2016)17mean patient age was 67.3 years $( \pm 9.5$ years), i.e. most of cases were observed in elder age group of the population.

Most of these $(61.2 \%)$ patients were male and only 31 patients $(38.8 \%)$ were females. Veronika Gagovic (2012)14out of 144 patients 73 were male
(51\%) Faming Zhang (2016)15also observed the similar observation in the study of 241 patients 133 male (55.19\%) Yang et al (2015)16 $80.95 \%$ were males, Robert A et al (2016)1762\% males i.e. male preponderance was observed in cases of pancreatic cancer.

Most of the female (45.2\%) as well as male (42.9\%) patients were in the age group of $51-60$ years followed by $41-50$ years $(32.3 \%$ of females and $24.5 \%$ of males respectively). No significant difference was seen in the age distribution of pancreatic mass among male and female subjects $(\mathrm{p}=0.538)$.

In our study, most of the benign pancreatic lesions were found in 51- 60 years $(46.1 \%)$ and $41-50$ years $(38.5 \%)$ age group. Most of the malignant pancreatic lesions were also found in $51-60$ years $(43.2 \%)$ and $41-50$ years $(25.4 \%)$. No significant difference was found in the age distribution of benign and malignant pancreatic lesions $(\mathrm{P}=0.754)$.

Veronika Gagovic (2012)14 There was no significant difference in age $(\mathrm{P}=0.0675)$ or sex $(\mathrm{P}=0.3595)$ between patients who had adenocarcinoma versus NPPA.

In our study patients presenting with solid pancraetic lesion found that $67 \%$ of the lesions were located in head of the pancreas, $15 \%$ were located in the uncinate, $13 \%$ were located in the body and $5 \%$ were located in the tail. 
According to Mohamad A. Eloubeidi et al (2003)18, in evaluating the yield of EUS-FNAB in the diagnosis of patients presenting with solid pancreatic lesions found that $65 \%$ of the lesions were situated in the head of pancreas, $12 \%$ of it were located in the uncinate, $17 \%$ in the body and $6 \%$ in the tail.

According to Guilia A Zamboni et al (2009)19, 63.0\% of the lesions were located in head or uncinate process of the pancreas and $35.2 \%$ of the lesions were located in the body or tail of the pancreas. Among these lesions ultrasound-guided fine-needle aspiration cytologic sampling had $99.4 \%$ sensitivity, 100\% specificity, and $99.4 \%$ accuracy.

EUS - FNAB suggested malignant lesion in most patients $(68.8 \%)$ while benign lesion was suggested in $26.2 \%$ patients. Four $(5 \%)$ patients had suspicious findings on EUS - FNAB.

Final diagnosis was made on basis of the histopathology findings. Most of the patients $(83.8 \%)$ were finally diagnosed with malignant pancreatic mass and only 13 (16.2\%) were diagnosed with benign pancreatic mass. Of the 4 suspicious lesions 3 turned out to be malignant and only 1 was benign.

According to Sean D. Paulsen et al (2005)20, 92 of 107 masses analyzed to have true-positive results. Histopathologic analysis of the core samples revealed 76 biopsy samples sufficient for a diagnosis of pancreatic ductal adenocarcinoma

M Vossetal (2000)21EUS-FNAB was feasible in 90 patients (adenocarcinomas, $\mathrm{n}=59$; neuroendocrine tumours, $\mathrm{n}=15$; various neoplasms, $\mathrm{n}=6$; pancreatitis, $\mathrm{n}=10$ ), and analysable material was obtained in 73.

Robert A et (2016)17the cumulative yield after repeat EUS-FNA for definite pancreatic adenocarcinoma was 7 (16\%).

Faming Zhang (2016)15 Pancreatic adenocarcinoma was the final diagnosis in $87.6 \%$ of patients.

Out of the 67 patients with malignant mass, 55 $(82.1 \%)$ patients were correctly diagnosed by EUS FNAB as malignant, while $9(13.4 \%)$ were wrongly suggested as benign by EUS - FNAB and out of the 4 suspicious lesion 3 were malignant on final diagnosis. Out of 13 patients with benign pancreatic mass, $12(92.3 \%)$ were correctly diagnosed to have benign lesion and one patient had suspicious finding. Diagnostic yield of EUS - FNAB is considering suspicious result as non-diagnostic in patients with Solid Pancreatic Masses. Specificity was found to be $100 \%$ Sensitivity of $82.09 \%$ PPV of $100 \%$ NPV was found to be low 52\%. This implicates that if EUS FNAB finding suggests benign lesion, the patients need to be cautiously followed up as there is $48 \%$ chance that the lesion may turn up to be malignant. The overall diagnostic accuracy of EUS FNAB was found to be $88.5 \%$ i.e. $88.5 \%$ of pancreatic masses are correctly classified as malignant / benign by EUS FNAB.

Diagnostic yield of EUS - FNAB is considering suspicious result as diagnostic in Patients with Solid Pancreatic Masses where Sensitivity of $86.57 \%$ Specificity $92.31 \%$ and PPV of $98.31 \%$.

According to Mohamad A. Eloubeidi et al (2003)18, of all the solid pancreatic masses, 72 yielded true positive results, 23 yielded true-negative results, and 4 yielded false-negative results. Hence, sensitivity, specificity, PPV, and NPV of EUS-FNAB for pancreatic solid masses were $94.7 \%$ (95\% CI, 89.7$99.8 \%$ ), $100 \%, 100 \%$, and $85.2 \%$ (95\% CI, $71.8-$ $98.6 \%$ ), respectively.

According to Gavin C Harewood et al (2002)22, FNA had $90 \%$ sensitivity for malignancy, $50 \%$ specificity for benign disease and $84 \%$ accuracy. Similarly among 36 patients with negative ERCP tissue samplingresults for EUS FNA which were $94 \%, 67 \%$ and $92 \%$ respectively.

According to Sukru Mehmet Erturk et al (2005)23, Among small masses, the diagnostic rate and sensitivity for biopsies guided using CT (100\% and $100 \%$, respectively) were not significantly different from those for biopsies guided using endoscopic sonography ( $90.9 \%$ and $93.8 \%$, respectively). For large masses, the diagnostic rate \& sensitivity $(96.6 \%$ \& $92.3 \%$ respectively) for biopsies guided using CT were not significantly different from those for biopsies guided using endoscopic sonography $(83.3 \%$ and $50 \%$, respectively). 
According to P. Thomas Cherian et al (2010)24, there were 78 pancreatic lesions, of which 65 were true positives (TP), 11 true negatives (TN) and two FN, giving an overall accuracy of $97 \%$ (76/78). Of nine periampullary lesions, 2 were TP, 6 were TN and 1 was $\mathrm{FN}$, giving an overall accuracy of $89 \%$ (8/9). The sensitivity, specificity, positive predictive value (PPV), negative predictive value (NPV) and accuracy of EUS-FNA for pancreatic and periampullary lesions combined were $96 \%, 100 \%$, $100 \%$ [95\% confidence interval (CI) 95-100\%], $85 \%$ (95\% CI 62-97\%) and 97\%, respectively.

NPV was found to be low $(57.14 \%)$ i.e. among patients suggested to have benign lesion by EUS FNAB, only $57.14 \%$ were finally diagnosed to have benign lesion, and rest $42.86 \%$ were wrongly suggested as benign. This implicates that if EUS FNAB finding suggests benign lesion, the patients need to be cautiously followed up as there is $42.86 \%$ chance that the lesion may turn up to be malignant. The overall diagnostic accuracy of EUS FNAB was found to be $88.5 \%$ i.e. $88.5 \%$ of pancreatic masses are correctly classified as malignant / benign by EUS FNAB.

Our study observed that the adenocarcinoma was the most common diagnosis in solid pancreatic masses found in $40(50 \%)$ of patients. Most common benign finding was chronic pancreatitis found in $21(26.2 \%)$ patients. Neuroendocrine carcinoma was found in $5(6.3 \%)$ patients. Malignant epithelial and poorly differentiated metastatic carcinoma were reported in 3 (3.8\%) pancreatic masses. Anaplastic giant cell carcinoma and spindle cell neoplasm were found in only 1 patient each. Suspicious for malignant finding on FNAC was reported in 4 patients.

Similar observation was found in the study conducted by M Voss et al (2000)21, which found that EUS-FNAB was feasible in 90 patients (adenocarcinomas, as 59; neuroendocrine tumours, as 15; various neoplasms, as 6; pancreatitis, $\mathrm{n}=10$ ), and analyzable material was obtained in 73 . Tumour size ( $>$ or $<25 \mathrm{~mm}$ in diameter) did not influence the ability to obtain informative biopsy samples. Diagnostic accuracy was $74.4 \%$ (adenocarcinomas,
81.4\%; neuroendocrine tumours, 46.7\%; other lesions, 75\%; $\mathrm{p}<0.02$ ). Overall, the diagnostic yield in all 99 patients was $68 \%$.

According to Sean D. Paulsen et al (2005)20 who observed that 92/ 107 masses analyzed to have truepositive results. Histopathology analysis of the core samples revealed 76 biopsy samples sufficient for a diagnosis of pancreatic ductal adenocarcinoma. Benign biopsy findings cannot be used to exclude the presence of a neoplasm, and repetition of a biopsy should be considered if there is high clinical suspicion of malignancy.

Veronika Gagovic (2012)14there were 21 patients (11\%) with initial FNA suspicious for malignancy who required a second attempt at tissue acquisition via repeat EUS-FNA, EUS-guided core biopsy or confirmed pathology based on surgical resection specimen. Out of 21 patients, 11 confirmed NPPA neoplasms, while 10 as primary pancreatic adenocarcinoma.

In this study we observed that most of the benign pancreatic lesions were found in males (76.1\%). Malignant pancreatic lesions were found more in males $(58.2 \%)$ as compared to females $(41.8 \%)$. No significant difference was found in the gender distribution of benign and malignant pancreatic lesions $(\mathrm{p}=0.339)$.

Carlo Fabbri et al (2014)25 found that the overall sensitivity, specificity, positive predictive value, negative predictive value, and accuracy were $80 \%$, $100 \%, 100 \%, 40 \%$, and $82 \%$, respectively. They have suggested that EUS-FNB of small pancreatic lesions using a 22-gauge ProCore needle is effective and safe which supports our hypothesis that EUSFNB is highly useful in establishing the nature of small pancreatic lesions.

Alexandra Kalogeraki et al (2016)26EUS-FNAB shows the sensitivity, specificity and accuracy of EUS-FNAB for pancreatic lesions range from $64 \%$ to $94 \%, 71 \%$ to $100 \%$ and $78 \%$ to $95 \%$ respectively. In different studies retrieved from PUBMED database since last 5 years, the sensitivity, specificity, positive predictive value, negative predictive value and accuracy of EUS-FNA for pancreatic solid masses were reported to be as 78 to 
$95 \%, 75 \%-100 \%, 98 \%-100 \%, 46 \%-80 \%$ and $78 \%-$ $95 \%$, respectively. There was no improvement of the efficacy of EUS-FNA even though new equipment and procedures have been developed.

Mohamed Malak et al (2016)27 observed the Sensitivity, specificity and accuracy were $98.9 \%$, $93.3 \%$ and $98.1 \%$ for EUS-FNA.

Jeremy Wang et al (2018)28they found that there were no significant differences in sensitivity between EUS-FNA and CT-FNA specimens $(73.7 \%$ vs. $88.9 \%, p=0.33)$. They observed that EUSguided FNA is as sensitive as CT-guided FNA in diagnosing pancreatic NETs, but its main advantage is in the diagnosis of smaller pancreatic NETs in the head of the pancreas.

\section{Conclusion}

EUS-FNAB is a safe and highly effective method for securing tissue diagnosis in patients with suspected pancreatic carcinoma. Almost all patients with suspicious cytology were subsequently proven to harbor cancer in the current study. Newer strategies, such as the addition of ancillary studies (e.g., tumor markers), are needed to further improve the yield and minimize suspicious and falsenegative results. Patients with suspicious EUSFNAB aspirates warrant further clinical evaluation.

\section{Bibliography}

1. Brand B, Pfaff T, Binmoeller KF, et al. Endoscopic ultrasound for differential diagnosis of focal pancreatic lesions, confirmed by surgery. Scand J Gastroenterol. 2000;35: 1221- 1228.

2. Muller MF, Meyenberger C, Bertschinger $\mathrm{P}$, Schaer R, Marincek B. Pancreatic tumors: evaluation with endoscopic US, CT, and MR imaging. Radiology. 1994;190:745-751.

3. Yeo CJ, Camero JL, Lillemoe KD Pancreaticodudenectomy for cancer of the head of the pancreas. Ann Sur 1995;221:721-731

4. Suits J, Frazee R, Erickson RA. Endoscopic ultrasound and fine needle aspiration for the evaluation of pancreatic masses. Arch Surg. 1999;134:639-642; discussion 42-43.

5. Itoi T, Sofuni A, Itokawa F, Irisawa A, Khor CJ, Rerknimitr R. Current status of diagnostic endoscopic ultrasonography in the evaluation of pancreatic mass lesions. Dig Endosc. 2011;23Suppl 1:17-21.

6. Wallace MB, Kennedy $\mathrm{T}$, Durkalski V, Eloubeidi MA, Etamad R, Matsuda K, et al. Randomized controlled trial of EUS-guided fine needle aspiration techniques for the detection of malignant lymphadenopathy. GastrointestEndosc. 2001;54:441-7.

7. Turner MS, Goldsmith JD. Best practices in diagnostic immunohistochemistry: spindle cell neoplasms of the gastrointestinal tract. Arch Pathol Lab Med. 2009;133:370-4.

8. Siddiqui UD, Rossi F, Rosenthal LS, Padda MS, Murali-Dharan V, Aslanian HR. EUSguided FNA of solid pancreatic masses: a prospective, randomized trial comparing 22gauge and 25-gauge needles. Gastrointest Endosc. 2009; 70:1093-7.

9. Bhutani MS, Hawes RH, Baron PL, et al. Endoscopic ultrasound guided fine needle aspiration of malignant pancreatic lesions. Endoscopy. 1997;29:854-858.

10. Michael Jonathan Hewitt, Mcphail, Mrcp, Lucia Possamai, AmeetDhar, Panagiotis Vlavianos, Kevin J. Monahan. EUS-guided FNA for diagnosis of solid pancreatic neoplasms: a meta-analysis. Gastrointestinal Endoscopy. 2012;75(2):319-31.

11. Niederhuber Je, Murray F. Brennan, Herman R. Menck. The national cancer data base report on pancreatic cancer. Cancer. 1995;76(9):1671-77.

12. Cleveland P, Gill KR, Coe SG, Woodward TA, Raimondo M, Jamil L, et al. An evaluation of risk factors for inadequate cytology in EUS-guided FNA of pancreatic tumors and lymph nodes. Gastrointest Endosc. 2010;71:1194-9.

13. Beckingham IJ. ABC Of Liver, pancreas and gallbladder, 1st ed. London: BMJ: 2001 
14. Veronika Gagovic, Bret J Spier, Ryan J DeLee, Courtney Barancin, Mary Lindstrom Michael Einstein MD, Siobhan Byrne, Josephine Harter MD, Rashmi Agni, Patrick R Pfau, Endoscopic ultrasound fine-needle aspiration characteristics of primary adenocarcinoma versus other malignant neoplasms of the pancreas, Can J Gastroenterol 2012;26(10):691-696.

15. Faming Zhang, Vivek Kumbhari, Alan H Tieu, Mohamad H E Zein, Ahmed Messallam, Vikesh K Singh, Anne Marie Lennon, Marcia I Canto, Anthony N Kalloo, Mouen A Khashab, Endoscopic UltrasoundGuided Fine Needle Aspiration of Suspected; Pancreatic Adenocarcinoma: Yield of the First and RepeatJ Pancreas. 2016 Jan 08; 17(1):48-52.

16. Min Jae Yang et al (2015) Min Jae Yang, HyuneeYim, Jae Chul Hwang, Dakeun Lee, Young Bae Kim, Sun Gyo Lim, Soon Sun Kim, Joon Koo Kang, Byung Moo Yoo and Jin Hong Kim, Endoscopic ultrasoundguided sampling of solid pancreatic masses: 22-gauge aspiration versus 25-gauge biopsy needles. BMC Gastroenterology (2015) 15:122.

17. Robert A et (2016)Robert A. Mitchell, Dylan Stanger, Constantin Shuster, Jennifer Telford, Eric Lam, and Robert Enns, Repeat Endoscopic Ultrasound-Guided Fine-Needle Aspiration in Patients with Suspected Pancreatic Cancer: Diagnostic Yield and Associated Change in Access to Appropriate Care, Canadian Journal of Gastroenterology and Hepatology; 2016: 7.

18. Robert A et (2016)Robert A. Mitchell, Dylan Stanger, Constantin Shuster, Jennifer Telford, Eric Lam, and Robert Enns, Repeat Endoscopic Ultrasound-Guided Fine-Needle Aspiration in Patients with Suspected Pancreatic Cancer: Diagnostic Yield and Associated Change in Access to Appropriate Care, Canadian Journal of Gastroenterology and Hepatology; 2016: 7.
19. Giulia A. Zamboni1, Mirko D’Onofrio1, Antonio Idili1, Roberto Malagò1, Roberta Iozzia1, Erminia Manfrin2, Roberto Pozzi Mucelli1, Ultrasound-Guided Percutaneous Fine-Needle Aspiration of 545 Focal Pancreatic Lesions December 2009.

20. Sean D. Paulsen et al (2005) Sean D. Paulsen Hanh V. Nghiem Edsa Negussie Ellen J. Higgins Elaine M. Caoili Isaac R. Francis Paulsen SD, Nghiem HV, Evaluation of Imaging-Guided Core Biopsy of Pancreatic Masses, AJR: 187, September 2006.

21. M Voss, P Hammel, G Molas, L Palazzo, A Dancour, D O'Toole, B Terris, C Degott, P Bernades, $\mathrm{P}$ Ruszniewski, Value of endoscopic ultrasound guided fine needle aspiration biopsy in the diagnosis of solid pancreatic masses.Gut 2000;46:244-249

22. C Harewood, Gavin, J Wiersema, Maurits. Endosonography-guided fine needle aspiration biopsy in the evaluation of pancreatic masses. The American journal of gastroenterology2002.

23. Sukru Mehmet Erturket al (2005) Guidance Sukru Mehmet Erturk Koenraad J. Mortelé Kemal Tuncali John R. Saltzman Roger Lao Stuart G. Silverman, Fine-Needle Aspiration Biopsy of Solid Pancreatic Masses: Comparison of CT and Endoscopic Sonography. AJR:187, December 2006.

24. P. Thomas Cherian, Prasoon Mohan, Abdel Douiri, Philippe Taniere, Rahul K. Hejmadi $\&$ Brinder S. Mahon Role of endoscopic ultrasound-guided fine-needle aspiration in the diagnosis of solid pancreatic and peripancreatic lesions: is onsite cytopathology necessary? International Hepato-Pancreato-Biliary Association HPB 2010, 12, 389-395.

25. Carlo Fabbri, Yasuda I, et al. Low diagnostic yield of transduodenal endoscopic ultrasound-guided fine needle biopsy using the 19-gauge Flex needle: A large 
multicenter prospective study. Endosc

Ultrasound. 2017;6(6):402-408.

26. Alexandra Kalogeraki, Georgios Z.

Papadakis, DimitriosTamiolakis, Iliana

Karvela-Kalogeraki, MihailosKarvelas-

Kalogerakis, John Segredakis, Michael

Papadakis, EleniMoustou, GalateiaDatseri,

Maria Tzardi, EUS - Fine- Needle

Aspiration Biopsy (FNAB) in the Diagnosis

of Pancreatic Adenocarcinoma: A Review. J.

Intern. Med., 2016, 54, 1, 24-30.

27. Malak M, Masuda D, Ogura T, ImotoA, Abdelaal UM, Sabet EA, Abo Dahab LH, Higuchi KYield of endoscopic ultrasoundguided fine needle aspiration and endoscopic retrograde cholangiopancreatography for solid pancreatic neoplasms. Scand J Gastroenterol. 2016 Mar;51(3):360-7.

28. Jeremy Wang, Surbhi Jain, Dion Chen, Wei Song, Chi-Tan $\mathrm{Hu}$, and Ying-Hsiu $\mathrm{Su}$, Development and Evaluation of Novel Statistical Methods in Urine BiomarkerBased Hepatocellular Carcinoma Screening. Sci Rep. 2018; 8: 3799. 